\title{
Female patients with rheumatoid arthritis - hospitalisation costs
}

\author{
Luana Andreea Macovei ${ }^{1,2}$, Maria Alexandra Burlui ${ }^{1,2}$, Anca Cardoneanu ${ }^{1,2}$, \\ Carmen Marinela Cumpat ${ }^{2}$, Elena Rezus ${ }^{1,2}$ \\ ${ }^{1}$ Department of Rheumatology, "Grigore T. Popa" University of Medicine and Pharmacy, lasi, Romania \\ ${ }^{2}$ Ist Rheumatology Clinic, Clinical Rehabilitation Hospital, Iasi, Romania
}

\begin{abstract}
Objective. Rheumatoid arthritis (RA) has direct costs, expressed as hospitalization, tests, drug therapy, physical therapy, assistance, transportation and equipment, and indirect costs caused by work loss. The aim of our study was to evaluate the direct costs of hospitalization in a Romanian clinical setting.

Methods. Relationships between the clinical, laboratory, functional parameters and the hospitalization costs were assessed in 50 female RA patients admitted to the Ist Rheumatology Clinic between May and July 2019. The duration of hospitalization ranged between 4 and 11 days.

Results. Our study showed that pain was associated with higher hospitalization costs, as the number of painful joints $(r=+0.396, p=0.004)$ and the Visual Analogue Scale for Pain $(r=+0.318, p=0.025)$ were significantly correlated with the total costs of hospitalization. A higher DAS28 required higher medication costs $(r=+0.434, p$ $=0.002)$ and the laboratory exams were more expensive in CRP tests $(r=+0.282, p=0.047)$.

Conclusions. Our study showed that in Romania there are high hospitalization costs for RA. These costs have exceeded per week the 2019 average monthly net salary of $\sim 3,000$ RON.
\end{abstract}

Keywords: rheumatoid arthritis, hospital costs, economic burden of disease, reimbursement

\section{INTRODUCTION}

Rheumatoid arthritis (RA) involves high healthcare costs because it is a chronic disease with long term treatments. The use of biologic therapy has largely increased the financial burden of RA, as they are more expensive than conventional drugs and represent approximately $90 \%$ of overall costs $(1,2)$. There is a range in prices paid for biologic agents, from the less expensive etanercept, rituximab and tocilizumab, to the medium-priced adalimumab and the most expensive infliximab and abatacept. Differences in costs may arise from changes in the approved maximum dose. Changes in patient weight affect the costs in drugs dosed based on weight, such as tocilizumab, infliximab and abatacept. Even if the costs of biological therapy have continuously decreased since 2009 with increased prescription volumes, its use in daily practice in Europe has led to a 3 - to 6-fold increase in direct costs (3). However, the rise of costs was compensated for by shorter sick leave and hospital stay $(4,5)$.

Direct costs encompass also the comorbidities $(6,7)$. Another financial impact can be found in wages of carers and laboratory measurements such as complete blood count test, the tuberculin skin test or Quantiferon tb Gold test, the hepatitis B antibody, metabolic and hepatic function tests $(1,8)$.

In Romania, costs of RA care can be estimated based on reimbursement data from the National Health Insurance House. The Romanian health care system provides universal coverage with basic health services which are financed by taxes and operate mainly within the public sector (9). Out-of-pocket expenses involving prescription drugs influence patient compliance to RA treatment (10) and exacerbate socioeconomic inequalities driven by an ageing population affected by emigration as there is in Romania. Nevertheless, it is difficult to estimate the 
costs per patient based on the general costs provided by the National Health Insurance House, due to differences in the regional and hospital specific prices (e.g. private practice $v s$. public service).

Romanian RA patients have recently participated in BRASS - Burden of Rheumatoid Arthritis across Europe Socioeconomic Survey, a multicenter observational study on the impact of disease duration, disease activity expressed as DAS28-CRP scores and pain level on professional life in ten European countries: UK, Spain, Germany, Italy, France, Denmark, Sweden, Hungary, Poland and Romania. BRASS shows that pain plays an important role in losing the ability to work, which leads to overall economic loss (11).

Repeated hospitalizations and the larger number of medical visits than required in other rheumatic diseases lead to higher hospitalization expenses in RA patients.

\section{MATERIAL AND METHODS}

Clinical, laboratory and functional parameters will be correlated with hospitalization costs in $50 \mathrm{fe}$ male RA patients admitted to the I ${ }^{\text {st }}$ Rheumatology Clinic of the Clinical Rehabilitation Hospital Iasi between May and July 2019. In our study group, the number of hospitalization days averaged 8.92, with a minimum of 4 days and a maximum of 11 days. All patients were assessed by a team made of rheumatologists, laboratory professionals, physiotherapists and nurses.

Inclusion criteria were age $\geq 18$ years, a definite RA diagnosis, a stable therapeutic regimen for at least 6 months prior to hospital admission, signing of informed consent forms. Exclusion criteria included age $<18$ years, overlap syndromes and patients' refusal to participate in the study.

The data were extracted from the patients' health records and standardized forms were filled out. Statistical data analysis was performed with IBM SPSS Statistics v20. Subgroup differences were investigated using nonparametric tests (Mann-Whitney and Kruskal-Wallis). We assessed the correlations between various variables with the Pearson coefficient, using a statistical significance threshold of $p<0.05$.

\section{RESULTS}

Costs are presented in year-2019 Romanian Leu (RON). Only direct costs contributed to the calculat- ed costs. The most used disease-modifying antirheumatic drug (DMARD) administered during the study period was methotrexate, but the costs of this drug and of biological agents were not included in our cost assessment because they are financed by the National Health Insurance House. Therefore, medication costs apply to nonsteroidal anti-inflammatory drugs (NSAIDs), glucocorticoids, analgesics and medication for iron deficiency anemia.

The total cost correlated significantly with the number of days of hospitalization $(\mathrm{r}=+0.939$; $p=0.001)$, the number of painful joints $(\mathrm{r}=+0.396$, $p=0.004)$ and Visual Analogue Scale for Pain VASp $(\mathrm{r}=+0,318, p=0.025)$.

Medication costs correlated significantly with $\mathrm{Hb}$ $(\mathrm{r}=-0.396, p=0.004)$, erythrocyte sedimentation rate $\operatorname{ESR}(\mathrm{r}=+0.476, p=0.001)$, C-reactive protein $\mathrm{CRP}(\mathrm{r}=+0.378, p=0.007)$, Visual Analogue Scale VAS $(\mathrm{r}=+0,286, p=0.044)$ and Disease Activity Score 28 DAS28-CRP $(\mathrm{r}=+0.434, p=0.002)$.

The laboratory costs correlated significantly with the levels of C-reactive protein CRP $(\mathrm{r}=+0.282, p$ $=0.047$ ).

The number of painful joints, the number of swollen joints and pain intensity on the VAS scale influence the total expenses of hospitalization in RA patients.

As expected, the disease activity score DAS28-CRP correlated significantly with the medication prescribed during hospitalization. Drug costs correlate with the level of disability, with an increase of costs from patients with good functional capacity to patients with mild, moderate, and severe functional disability.

The inflammatory syndrome is directly related to medication costs, but only CRP correlates with the costs of laboratory tests.

\section{DISCUSSIONS}

The mean annual cost of RA was used in many meta-analyses and systematic reviews. Instead of focusing on annual expenditures, our study aims at estimating the hospital burden associated with RA treatment, so that cost-effective measures can be made and adjusted to local circumstances. The absenteeism and productivity loss were beyond the scope of our study. Therefore, payments for sick leave of salaried workers, allowances associated with permanent disability and help provided by family and friends for household and outdoor activities were not considered in our analysis of RA costs. 
Cost-related lack of therapy may concern not only individuals, but also healthcare facilities. All patients had public healthcare insurance and no private healthcare insurance was used. Therefore, some restrictive treatment and administrative guidelines associated with the Romanian Gross Domestic Product, ranked 15th in the European Union in 2018, and the reticence towards private funding should be also considered. Copayment was defined in Romania only as a financial support for private hospitals provided by National Health Insurance House. Socioeconomic difficulties faced by hospitals are related to direct costs which involve examinations, laboratory tests, hospital admission, conventional synthetic and biologic disease modifying drugs, caregiver assistance, transportation and equipment. The improvement in RA symptoms through rheumatologist and nurse-led care (12), the use of conventional DMARDs instead of the more expensive biological therapy (13), the use of subcutaneous-injected biologic agents instead of the more expensive infused
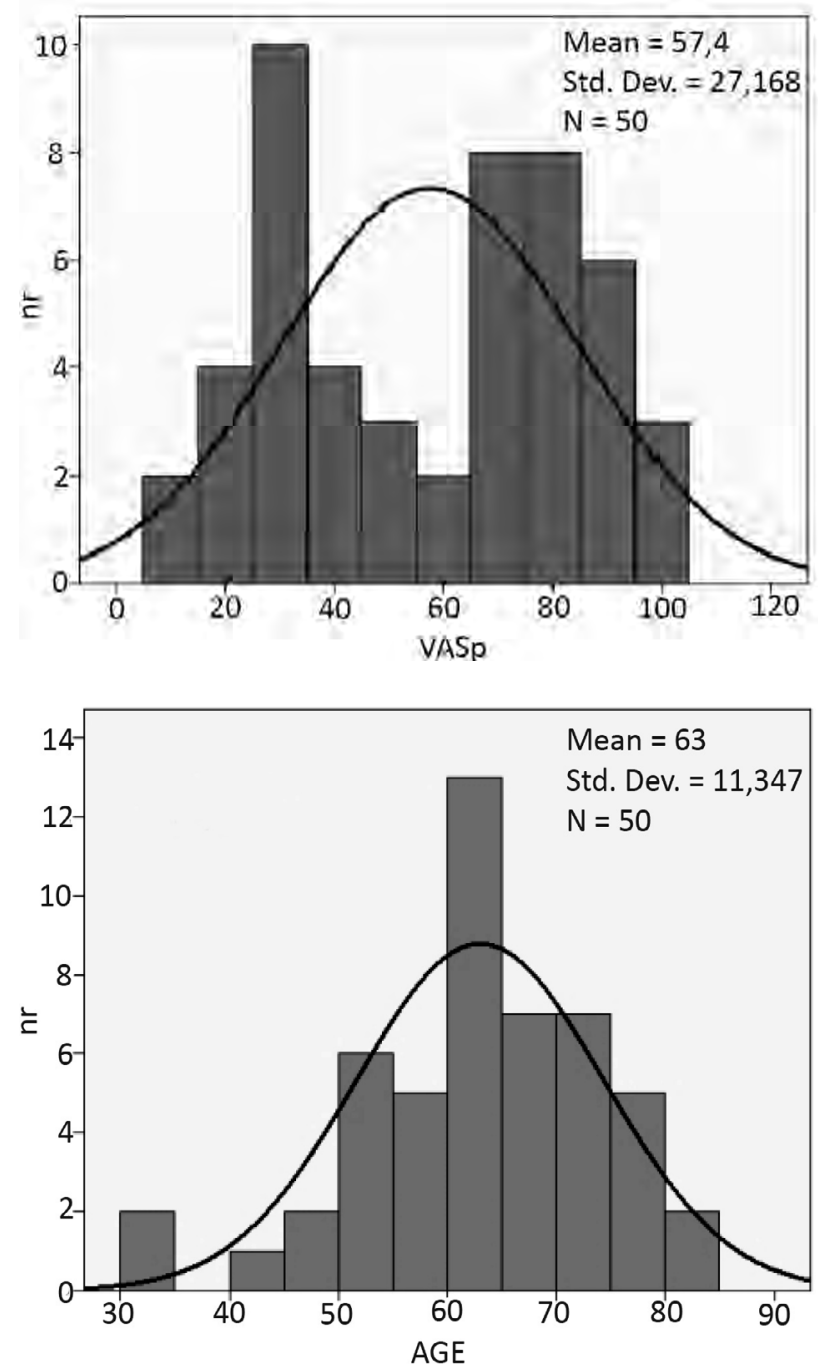

biologic therapy, a larger clinical setting with high patient volume involving a more efficient use of equipment (1), avoiding switching to another anti-TNF or to a biologic with a different mechanism of action (14), earlier treatment initiation, improved treatment strategies with conventional DMARDs, including triple therapy (3), negotiated cost discounts and utilization limits $(15,16)$ can be used for cost optimization.

Hospital care is more expensive than the procedures performed at a physician's office, an outpatient center or at home $(17,18)$. The financial advantages of home care in Romanian RA patients needs further investigation, but the intangible costs related to quality of life for both patients and families should also be considered in such analyses.

In RA patients, medical expenses have shifted from hospital care in the 1980s and 1990s towards drug costs over more recent decades, due to the high price of biological agents $(19,20)$. The use of biological agents has increased costs 3 to 6 -fold $(21,22)$.

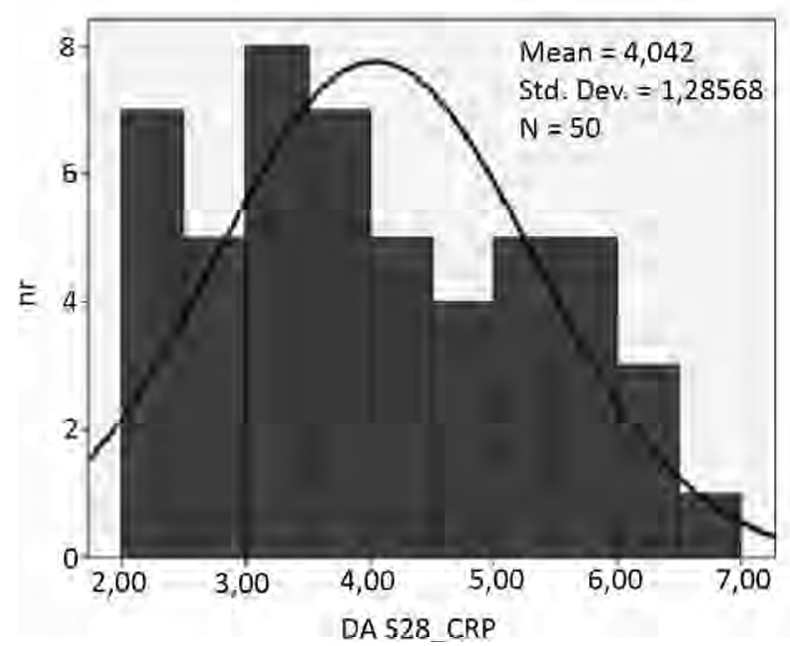

FIGURE 1. Case distribution according to the VAS pain scale, DAS28-CRP and age 

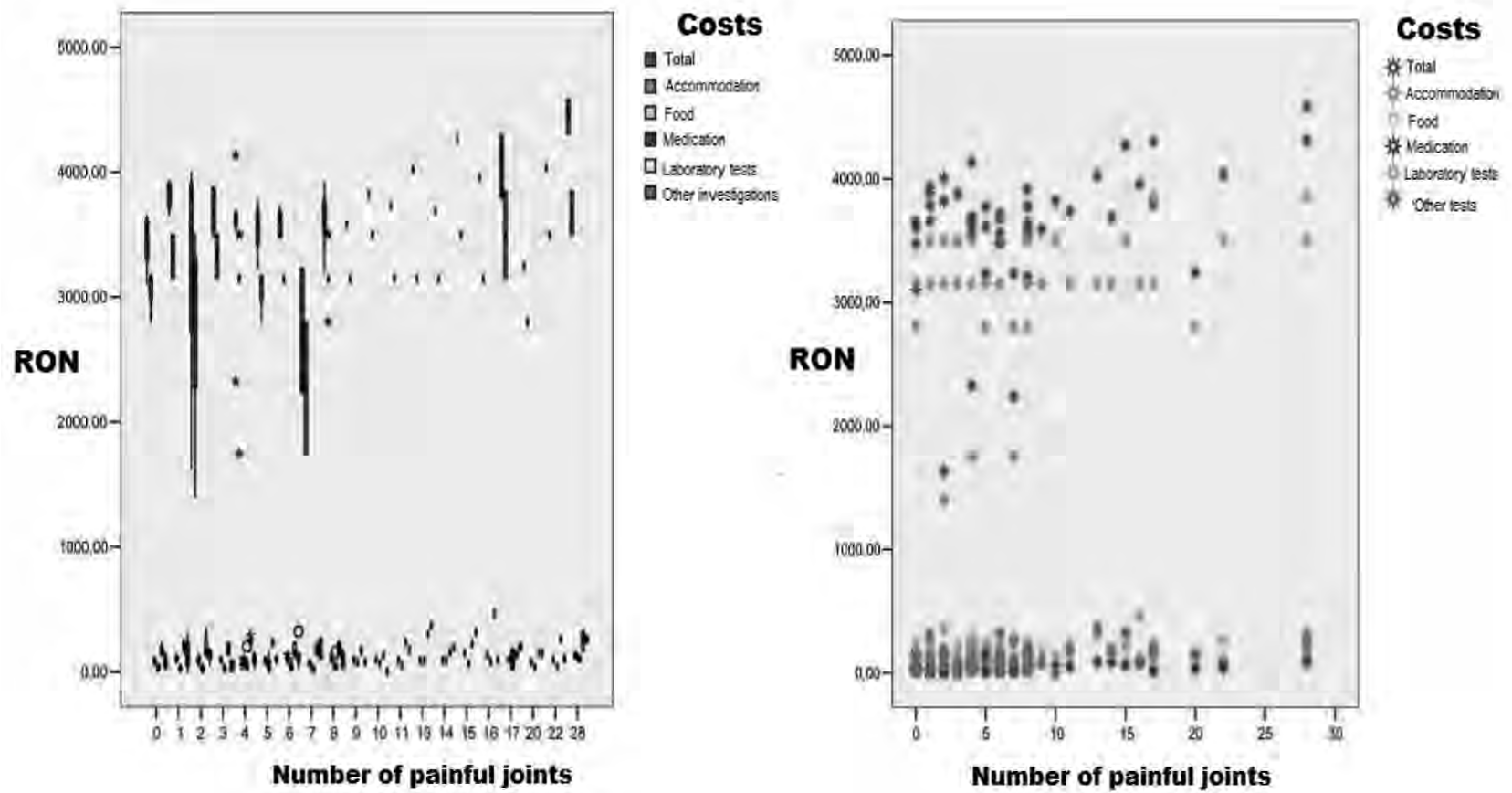

FIGURE 2. Various costs expressed in RON associated with the number of painful joints

The costs described in our study were targeted at covering hospital stay, medication, food, laboratory tests and other investigations. Drug reimbursement addressed management of both RA and comorbidities. In our study group, pain, which is associated with increased hospitalization costs, was the most common cause of referral.

TABLE 1. Hospitalization costs expressed as RON in a group of female RA patients

\begin{tabular}{|l|l|l|l|}
\hline Domain & median & minimum & maximum \\
\hline $\begin{array}{l}\text { Number of days of } \\
\text { hospitalization }\end{array}$ & 8.92 & 4 & 11 \\
\hline ESR (Normal values = 4-23) & 38 & 1 & 136 \\
\hline CRP (Normal values = 0-1) & 1.98 & 0.01 & 12.30 \\
\hline Number of painful joints & 7.54 & 0 & 28 \\
\hline Number of swollen joints & 1.58 & 0 & 13 \\
\hline VAS pain (0-100) & 27 & 10 & 100 \\
\hline DAS28-CRP & 4.04 & 2.09 & 6.73 \\
\hline $\begin{array}{l}\text { Total reimbursement } \\
\text { (RON) }\end{array}$ & 3624 & 1632 & 4585 \\
\hline $\begin{array}{l}\text { Reimbursement for hospital } \\
\text { stay (RON) }\end{array}$ & 3122 & 1400 & 3850 \\
\hline Food reimbursement (RON) & 96 & 40 & 150 \\
\hline Drug reimbursement (RON) & 54 & 0 & 204 \\
\hline $\begin{array}{l}\text { Laboratory reimbursement } \\
\text { (RON) }\end{array}$ & 206 & 133 & 465 \\
\hline $\begin{array}{l}\text { Reimbursement for further } \\
\text { investigations (RON) }\end{array}$ & 128 & 0 & 370 \\
\hline
\end{tabular}

Our study group included exclusively female patients. With a mean age of 63 years, mostly retired women were involved, so that indirect costs caused by productivity loss were irrelevant.

Clinical data included acute phase reactants such as erythrocyte sedimentation rate (ESR), C-reactive protein (CRP), disease activity score (DAS) and visual analog scales (VAS).

The duration of hospitalization ranging from 4 to 11 days was not influenced by adverse effects of medication, as no patient in our study group has experienced serious side effects.

\section{CONCLUSIONS}

Adapting treatment to the patients' needs and providing counseling to patients for a better knowledge of the disease may reduce unnecessary medical visits and tests.

Prior to initiation of biological therapy, rheumatologists should carefully consider the benefits for the patient, so that savings can be made through low cost therapies. Another long term solution is to integrate RA patients within community-based services instead of hospitals, given the high hospitalization costs, which in our study have exceeded per week the 2019 average monthly net salary of $\sim 3,000$ RON.

Conflict of interest: none declared Financial support: none declared 


\section{REFERENCES}

1. Schmier J, Ogden $\mathrm{K}$, Nickman N, et al. Costs of providing infusion therapy for rheumatoid arthritis in a hospital-based infusion center setting. Clinical therapeutics. 2017 Aug 1;39(8):1600-17.

2. Cross M, Smith E, Hoy D, et al. The global burden of rheumatoid arthritis: estimates from the global burden of disease 2010 study. Ann Rheum Dis. 2014;73(7):1316-1322.

3. Huscher D, Mittendorf T, von Hinüber U, et al., German Collaborative Arthritis Centres. Evolution of cost structures in rheumatoid arthritis over the past decade. Annals of the rheumatic diseases. 2015 Apr 1;74(4):738-45.

4. Eriksson JK, Johansson K, Askling J, et al. Costs for hospital care, drugs and lost work days in incident and prevalent rheumatoid arthritis: How large, and how are they distributed? Annals of the rheumatic diseases. $2015 \mathrm{Apr}$ 1;74(4):648-54.

5. Sugiyama N, Kawahito Y, Fujii T, et al. Treatment patterns, direct cost of biologics, and direct medical costs for rheumatoid arthritis patients: a real-world analysis of nationwide Japanese claims data. Clinical therapeutics. 2016 Jun 1;38(6):1359-75.

6. Tănăsescu $C$, Ionescu RA. Chronic hepatitis $C$ virus infection mimicking rheumatoid arthritis. Romanian Journal of Internal Medicine. 2003;41(2):205-11.

7. Han GM, Han XF. Comorbid conditions are associated with healthcare utilization, medical charges and mortality of patients with rheumatoid arthritis. Clinical rheumatology. 2016 Jun 1; 35(6):1483-92.

8. Uhlig T, Moe RH, Kvien TK. The burden of disease in rheumatoid arthritis. Pharmacoeconomics. 2014 Sep 1;32(9):841-51.

9. Vlădescu C, Scîntee G, Olsavszky V. Romania: Health system review. Health systems in transition. 2008;10(3):1-181.

10. Heidari P, Cross W, Crawford K. Do out-of-pocket costs affect medication adherence in adults with rheumatoid arthritis? A systematic review. InSeminars in arthritis and rheumatism 2018 Aug 1;48(1):12-21.

11. Galloway J, Capron JP, Leonardis FD, et al. Summary of findings from the Burden of Rheumatoid Arthritis across Europe Socioeconomic Survey (BRASS). Rheumatology. 2019 Apr 1;58(Supplement_3):kez106-074.

12. Wang J, Zou X, Cong L, et al. Clinical effectiveness and costeffectiveness of nurse-led care in Chinese patients with rheumatoid arthritis: A randomized trial comparing with rheumatologist-led care.
International journal of nursing practice. $2018 \mathrm{Feb}$;24(1):e12605.

13. Santos-Moreno P, Peralta LV, Cabrera M, et al. Use of an algorithm within a multidisciplinary consultation for the management of patients with rheumatoid arthritis candidates for biological therapy-a strategy for optimizing costs. Value in Health Regional Issues. 2019 Oct 1;19:S52.

14. Vanderpoel J, Tkacz J, Brady BL, et al. Health Care Resource Utilization and Costs Associated With Switching Biologics in Rheumatoid Arthritis. Clinical therapeutics. 2019 Jun 1; 41(6):1080-9.

15. Park PW, Dryer RD, Hegeman-Dingle R, et al. Cost burden of chronic pain patients in a large integrated delivery system in the United States. Pain Practice. 2016 Nov;16(8):1001-11.

16. Rai SK, Aviña-Zubieta JA, McCormick N, et al. Trends in gout and rheumatoid arthritis hospitalizations in Canada from 2000 to 2011. Arthritis care \& research. 2017 May;69(5):758-62.

17. Laires PA, Mesquita R, Veloso L, et al. Patient's access to healthcare and treatment in rheumatoid arthritis: the views of stakeholders in Portugal. BMC musculoskeletal disorders. 2013 Dec;14(1):279.

18. Hresko A, Lin TC, Solomon DH. Medical Care Costs Associated With Rheumatoid Arthritis in the US: A Systematic Literature Review and Meta-Analysis. Arthritis Care Res (Hoboken). 2018;70(10):1431-1438.

19. Fautrel B, Cukierman G, Joubert JM, et al. Healthcare service utilisation costs attributable to rheumatoid arthritis in France: analysis of a representative national claims database. Joint Bone Spine. 2016 Jan 1;83(1):53-6.

20. Husberg M, Davidson T, Hallert E. Non-medical costs during the first year after diagnosis in two cohorts of patients with early rheumatoid arthritis, enrolled 10 years apart. Clinical rheumatology. 2017 Mar 1;36(3):499-506.

21. Leon L, Abasolo L, Fernandez-Gutierrez B, et al. Direct medical costs and their predictors in the EMAR-II cohort: Variability in the management of rheumatoid arthritis and spondyloarthritis in Spain. Reumatología Clínica (English Edition). 2018 Jan 1;14(1):4-8.

22. Lim SY, Lu N, Oza A, et al. Trends in gout and rheumatoid arthritis hospitalizations in the United States, 1993-2011. JAMA. 2016 Jun 7;315(21):2345-7. 